\title{
Equilibrium abundances in hot DA white dwarfs as derived from self-consistent diffusion models
}

\section{Analysis of spectroscopic EUVE data}

\author{
S. L. Schuh ${ }^{1}$, S. Dreizler ${ }^{1}$, and B. Wolff ${ }^{2}$ \\ 1 Institut für Astronomie und Astrophysik (IAAT), Universität Tübingen, 72076, Tübingen, Germany \\ e-mail: schuh@astro.uni-tuebingen.de, dreizler@astro.uni-tuebingen.de \\ 2 Institut für Theoretische Physik und Astrophysik, Universität Kiel, 24098 Kiel, Germany \\ e-mail: wolff@astrophysik.uni-kiel.de
}

Received 5 June 2001 / Accepted 8 November 2001

\begin{abstract}
We present the first analysis of an EUV selected sample of hot DA white dwarfs using a new type of atmospheric models. These models take into account the interplay between gravitational settling and radiative acceleration to predict the chemical stratification from an equilibrium between the two forces while self-consistently solving for the atmospheric structure. In contrast to atmospheric models with the assumption of chemical homogeneity, the number of free parameters in the new models is reduced to the effective temperature and surface gravity alone. The overall good reproduction of observed EUV spectra reveals that these models are able to describe the physical conditions in hot DA white dwarf atmospheres correctly. A comparison with previous analyses highlights the improvements as well as the limits of our new models.
\end{abstract}

Key words. white dwarfs - stars: fundamental parameters - stars: abundances - stars: atmospheres - diffusion

\section{Introduction}

White dwarf (WD) atmospheres exhibit a quasi-monoelemental chemical composition. They are either practically pure hydrogen or pure helium, with the hydrogen rich sequence being called DA and the helium rich sequence being summarized as non-DA. In the following, we will restrict our discussion to DA white dwarfs, even though similar physics applies to non-DAs. The basic mechanism for purification of white dwarf envelopes was first explained by Schatzman (1949): the strong gravitational field (typically, $\log g=8$ in cgs units) on the surface of WDs yields a steep pressure gradient. Pressure driven diffusion separates the elements according to their atomic weight, which is generally referred to as gravitational settling or sedimentation.

In hot WDs (an approximate effective temperature range starts from $T_{\text {eff }} \approx 40000 \mathrm{~K}$ upwards), however, this diffusion process is disturbed by the radiation pressure that elements experience, depending on the overlap of their wavelength-dependent opacity with the maximum spectral flux. The overlap of the maximal spectral

\footnotetext{
Send offprint requests to: S. L. Schuh,

e-mail: schuh@astro.uni-tuebingen.de

* Based on observations made with the EUVE Satellite.
}

intensity with the region of maximal opacity yields a considerable radiative acceleration on heavier elements in WD atmospheres, provided the luminosity is high enough (Vauclair et al. 1979; Fontaine \& Michaud 1979). In the considered temperature range the luminosities are indeed still high (i.e. $L / L_{\odot}>1$ ). This is sufficient to sustain photospheric elements other than hydrogen with abundances no higher than $10^{-4}$ relative to hydrogen. In optical spectra of hot white dwarfs, these trace pollutants become directly visible only when great effort is put in the detection of individual lines (Dupuis et al. 2000). Through resonance lines in the UV and even more through the sheer number of lines in the EUV, however, these spectral ranges are strongly affected. The flux deficit caused by the additional opacity in the EUV as compared to pure hydrogen atmospheres, hinted at already by earlier data (Mewe et al. 1975; Hearn et al. 1976; Lampton et al. 1976; Margon et al. 1976; Shipman 1976), became more and more evident as the quality of observations improved with the HEAO(Einstein), EXOSAT and ROSAT satellites (Kahn et al. 1984; Petre et al. 1986; Jordan et al. 1987; Paerels \& Heise 1989; Barstow et al. 1993; Jordan et al. 1994; Wolff et al. 1996). While one EXOSAT (Vennes et al. 1989) and various ROSAT observations revealed the opacity to be mainly due to absorbers other than helium, observations 
with EUVE made possible a more detailed investigation of the nature of absorbers.

The most recent analyses of hot white dwarf EUVE spectra have been presented by Barstow et al. (1997) and Wolff et al. (1998). Using the latest non-LTE and LTE atmosphere models, both groups derived a metal mix scaling factor for each object in their respective WD sample. Barstow et al. use this single scaling factor per object to adjust metal abundances predicted for its combination of effective temperature and surface gravity by Chayer et al. (1995a,b), whereas Wolff et al. use relative metal abundances which they have derived for the standard star G 191-B2B as a typical metal mix and give the appropriate scaling factor that they call metallicity for each of their sample objects.

In the models used, the distribution of the metals was assumed to be homogeneous, i.e. abundances were treated as being the same in all atmospheric depths. But as the effects of sedimentation and radiative levitation lead to a chemical gradient, fitting the emergent flux of real, stratified WD atmospheres with homogeneous atmosphere models has its limits, and true consistency cannot be achieved. Therefore, attempts were made to include the impact of a chemical gradient via an ad hoc stratification: For iron in G 191-B2B (Barstow et al. 1999), which could successfully reproduce the EUVE spectrum of G 191-B2B, and for nitrogen in RE J1032+535 (Holberg et al. 1999).

As discussed above, the basic physical mechanisms leading to the stratification have long been known, so their actual modelling appears preferable to an ad hoc approach. Chayer et al. (1995a,b) were the first to calculate an extensive model grid for white dwarfs predicting the chemical stratification assuming equilibrium between gravitational settling and radiative levitation. An improvement has now been achieved through the introduction of self-consistent non-NLTE model atmospheres which account for the coupling between chemical stratification and the radiation field (Dreizler 1999). The successful application of these models to the EUVE spectrum of G 191-B2B (Dreizler \& Wolff 1999) has motivated a more systematic analysis of a larger sample of hot DA white dwarfs which we will present here.

The first paper on this investigation exploits EUVE spectra, further papers will deal with the UV and optical wavelength ranges and give detailed metal abundance pattern predictions. The availability of EUVE spectra defines, as in Wolff et al. (1998), the overall sample, which is more thoroughly introduced in Sect. 2. The observations and particular considerations concerning the interstellar medium are shortly described there as well. Sect. 3 outlines the principle of the model calculations and focuses on the characteristics of the model grid computed for comparison with the sample on hand. The methods and results from fitting the new class of theoretical spectra to the observations are presented in Sect. 4 . What implications these results involve, and why further efforts in this direction are both justified by the present success and at the same time necessary due to remaining puzzles, is being discussed in Sect. 5.

\section{EUVE: Observations of hot DA white dwarfs}

Generally speaking, the maximum spectral flux of hot WDs lies in the extreme ultraviolet (EUV). The fact that the main opacity of heavy elements at these temperatures tends to lie in the same range means that radiative acceleration can sustain traces of them in the atmosphere, but it also implies that the EUV is the best suited spectral range for the detection of these elements. Consequently, we start by surveying EUVE spectra, even though its instrument's spectral resolutions are not high enough to identify individual lines. Identification element by element is, however, not a necessary requirement with the new models: they impose no need for the use of a pre-defined metal mix, nor do they require the adjustment of e.g. a scaling factor, as the abundances are not free parameters but are derived from an equilibrium condition (see Sect. 3). As the EUV flux is very sensitive to the chemical composition of the photosphere, a comparison in this regime is extremely useful to test the predictions of the diffusion models. Interpreting the repeated analysis as a test case for the models, it is reasonable to start out by following previous investigations as closely as possible. For this reason, we chose to use the 26 DAs already analyzed by Wolff (1999).

\subsection{The EUV selected DA sample}

This sample comprises all hot $\left(T_{\text {eff }}>40000 \mathrm{~K}\right)$ DA white dwarfs with EUVE observations, which are mostly taken from the EUVE public archive (now available at the multimission data archive at STScI), and a few from own observations. The data sets and the reduction procedures applied are described elsewhere (Wolff et al. 1998; Wolff 1999); those publications also show the reduced spectra in the form used here, i.e. flux calibrated SW, MW and LW data combined to yield a single spectrum.

The effective temperatures of the sample stars lie above $T_{\text {eff }}=40000 \mathrm{~K}$, where photospheric metal abundances still are large enough to be detectable at all. Above $T_{\text {eff }}=70000 \mathrm{~K}$, mass loss effects might start to disturb the expected equilibrium between gravitational and radiative acceleration (Unglaub \& Bues 1998), but the sample contains no stars hotter than that. Wolff et al. (1998) have grouped the objects into four different categories. The first set consists of G 191-B2B like objects, where the high metallicity yields a steep flux drop towards short wavelengths, beyond the interstellar He II absorption edge. The second group comprehends GD 246 and similar objects which contain less photospheric metals, but still more than those in the third group whose spectra Wolff et al. could fit with pure hydrogen atmospheres. A fourth rather inhomogeneous set compiles the remaining objects. For details, especially on the effective temperature, surface gravity and metallicity region in parameter space covered by the different groups, we refer once more to Wolff (1999). 


\subsection{Treatment of the $I S M$}

As briefly remarked on before, the interstellar medium can considerably attenuate the stellar flux. For the EUV, the most important features are the H I, He I and He II boundfree ground state absorptions with edges at $911.7 \AA$, 504.3 $\AA$ and $227.8 \AA$, respectively. Again, this is being accounted for in the same way as in Wolff et al. (1998): the effect of given interstellar column densities on the theoretical fluxes is modelled and applied following Rumph et al. (1994). The derivation of H I column density depends on the effective temperature of the model used, and the He I and He II column densities on its absorber content. Due to the interweavement of $T_{\text {eff }}$ and $N(\mathrm{HI})$ and the fact that the Lyman edge lies outside the range observed by EUVE, Wolff et al. (1998) have started from optically determined effective temperatures (by Finley et al. 1997), and additionally have retained surface gravities from that analysis unchanged. In the present approach, we start from the effective temperatures from Wolff (1999) and adopt his H I column densities unalteredly whenever possible.

Even though the HeI and He II column densities have to be constantly modified during the fitting procedure depending on the assumed photospheric parameters, all interstellar contributions will in the following be treated as a secondary effect on the stellar light that can be dealt with independently of the analysis of the source itself, disregarding the fact that an appropriate correction can only be made after a set of photospheric parameters has been adopted. As the interstellar absorption is more important at longer wavelengths while the absorption by photospheric metals increases towards shorter wavelengths, the different contributions can partly be separated.

\section{Equilibrium ansatz: Chemically stratified model atmospheres}

Under the assumptions that no processes competing with diffusion and levitation are present and that diffusion timescales are short, the distribution of trace elements in a stellar atmosphere can be expected to take on an equilibrium state, as proposed by Chayer et al. (1995a,b). In such a situation, mean diffusion velocities would be zero at any location in the atmosphere, due to an exact balance of gravitational, radiative and electrical forces (when neglecting thermal and concentration diffusion). A more detailed formulation of this condition for polluting elements $(i)$ in a hydrogen (or helium) plasma (1) can be found elsewhere (Dreizler 1999). It yields, in a short outline,

$$
\begin{aligned}
m_{1} F_{1}-m_{i} F_{i}= & \left(A_{i}-A_{1}\right) m_{\mathrm{p}} g-\left(Z_{i}-Z_{1}\right) \mathrm{e} E \\
& -A_{i} m_{\mathrm{p}} g_{\mathrm{rad}, i} \\
= & 0,
\end{aligned}
$$

where $m_{i} F_{i}$ are the forces acting on element $(i), A_{i}$ are the atomic weights, $Z_{i}$ are the mean electrical charges, $E$ is the electrical field caused by the separation of electrons and ions, $m_{\mathrm{p}}$ is the proton mass, and $g_{\mathrm{rad}, i}$ is the radiative acceleration acting on element $(i)$. Using hydrostatic equilibrium and charge conservation, this leads to the simple term

$g_{\mathrm{rad}, i}=g_{\mathrm{eff}, i}$.

One immediately recognizes that requiring diffusion velocities to be zero is indeed the same as asking for the effects of gravitational settling and radiative levitation to cancel each other. Knowing that the above quantities stand for

$$
\begin{aligned}
& g_{\mathrm{eff}, i}:=\left(1-\frac{A_{1}\left(Z_{i}+1\right)}{A_{i}\left(Z_{1}+1\right)}\right) g \\
& g_{\mathrm{rad}, i}=\frac{1}{\rho_{i}} \frac{4 \pi}{c} \int_{0}^{\infty} \kappa_{\nu, i} H_{\nu} \mathrm{d} \nu
\end{aligned}
$$

where $\rho_{i}$ is the mass density of the element $(i), \kappa_{\nu, i}$ is the frequency dependent absorption coefficient which includes all contributions of this element at the frequency $\nu$, and $H_{\nu}$ is the Eddington flux, it furthermore becomes clear that $g_{\mathrm{rad}, i}=g_{\mathrm{eff}, i}$ can only be fulfilled by fixed values for $\rho_{i}$. This equilibrium solution for $\rho_{i}$ is depth dependent. For a plane parallel model atmosphere in hydrostatic equilibrium this means that equilibrium abundances are solely determined by the photospheric parameters $T_{\text {eff }}$ and $\log g$, in other words, the photospheric abundances are no longer free parameters. Calculating the equilibrium abundances inevitably leads to a chemically stratified atmosphere.

Chayer et al. (1995a,b) have presented extensive results of such calculations for white dwarfs. Unfortunately, their predicted equilibrium abundances were not quantitatively consistent with observations. This might have been partly due to the fact that the observations were analyzed with chemically homogeneous model atmospheres, meaning that homogeneous abundances were compared to predictions for stratified atmospheres. It is more probable yet an effect that arises because the feedback of the modified abundances on the radiation field was not being accounted for.

A self-consistent solution of the equilibrium condition and the atmospheric structure has recently been presented (Dreizler 1999). In addition to this newly introduced coupling, it is also a novelty that the calculations are being performed under non-LTE conditions. This has turned out to be a crucial point (Schuh 2000; Dreizler \& Schuh 2001). The code itself relies on an iteration scheme that alternates between the determination of new equilibrium abundances and the corresponding solution for the atmospheric structure, which is described in Werner \& Dreizler (1999).

\subsection{Scope of application}

It remains to be justified why the white dwarfs in our sample will presumably obey the presented equilibrium condition. Processes eligible to disturb or prevent its adjustment can be mass loss, accretion from the interstellar medium, convection or mixing through rotation. As explained by Unglaub \& Bues (1998), mass loss would 
have the effect of homogenizing a chemical stratification, but as has also been shown in the same paper, mass loss rates drop below the critical limit $\left(10^{-16} M_{\odot} / \mathrm{yr}\right)$ for DAs cooler than $T_{\text {eff }}=70000 \mathrm{~K}$ so that this phenomenon should not occur in any of the sample stars. Calculations of MacDonald (1992) that treat the interaction between accretion and the white dwarf wind reveal that WDs in our sample are not affected by accretion. It is prevented since $L_{\mathrm{WD}} / L_{\odot}>1$, so that at least a critical mass loss rate of $3 \times 10^{-18}$ to $10^{-21} M_{\odot} / \mathrm{yr}$ is sustained.

Convection would of course homogenize abundances, too. However, convective instability in the outer layers occurs only below $T_{\text {eff }}=12000 \mathrm{~K}$ for DAs, i.e. well below $T_{\text {eff }}=40000 \mathrm{~K}$. Rotation could lead to a mixing through meridional currents; but as most white dwarfs are very slow rotators (Heber et al. 1997; Koester et al. 1998), this is not likely to interfere either.

Even if it is left undisturbed, it takes an atmosphere a certain time to reach an equilibrium state. Due to the high surface gravities, diffusion time scales are of the order of months in the outer layers of white dwarfs (Koester 1989, based on diffusion coefficients by Paquette et al. 1986). This is nothing but an instant compared to evolution time scales $\left(\sim 10^{7} \mathrm{yr}\right)$, and this is the reason why DA atmospheres in the effective temperature range between $40000 \mathrm{~K}$ and $70000 \mathrm{~K}$ can be regarded to have their photospheric abundances set to equilibrium values at any time of their evolutionary stage. In the temperature range above $40000 \mathrm{~K}$ the flux maximum still lies in the EUV, i.e. in the same range where multiple lines of heavier elements provide substantial opacity, making radiative levitation an efficient mechanism for sustaining absorbers. It should in this context also be noted that the evaluation of the equilibrium abundances does not include the contributions of thermal and concentration diffusion, which have much longer time scales.

\subsection{The model grid}

The model grid spans the $T_{\text {eff }}$ and $\log g$ plane as suggested by the results from Wolff (1999): the effective temperatures cover a range from $38000-70000 \mathrm{~K}$ in $2000-3000 \mathrm{~K}$ steps, the logarithmic surface gravity a range from $7.2-8.3$ in $0.1-0.2$ dex steps. The grid is however not complete and concentrates around the previously found parameter combinations for the objects as listed in Table 2. We emphasize once more that the total absorber content, i.e. the entire depth-dependent distribution of each individual trace element, is solely defined by a models' $\left[T_{\text {eff }}, \log g\right]$ - combination, which makes $T_{\text {eff }}$ and $\log g$ the only free parameters. The elements included in the model calculations are the same as those used by Wolff et al. (1998) for the definition of their metal mix, which in turn are those that they were able to identify in HST GHRS data of the standard G 191-B2B. Other elements were assumed to be present in abundances too low to significantly contribute to the EUV opacity (however, stratification may well invalidate this assumption). While other elements than those used in Wolff's and this analysis have been identified in UV spectra of some of the sample stars, including G 191-B2B itself, the low abundances of $2.5 \times 10^{-8}$ for phosphorus and $3.2 \times 10^{-7}$ for sulfur, for example, as reported from the analysis of ORFEUS spectra by Vennes et al. (1996a), confirm that these element's opacities will presumably not contribute significantly to the total EUV opacity. To investigate the possible effects of several additional elements anyhow (which currently results in a dramatic increase of CPU time demand), further analyses will have to be conducted (see also Sect. 5.2). For this analysis, we aimed at being able to compare our results to a previous analysis in the EUV, as mentioned before. Consequently, the list of elements considered besides $\mathrm{H}$ reads $\mathrm{He}, \mathrm{C}, \mathrm{N}, \mathrm{O}, \mathrm{Si}$, $\mathrm{Fe}$ and Ni. Differences remain with regard to the number of atomic levels and with regard to LTE/non-LTE population of these levels. Details for the model calculations presented here are summarized in Table 1. Note that so far, the model atoms used for the solution of the atmospheric structure and for the solution of the equilibrium condition are identical, which implies that the relatively high accuracy required for the correct evaluation of the radiative acceleration slows down the atmospheric structure calculation.

The time it takes a model with the specified atomic data to converge depends primarily on the stratification of the input model. For homogeneous input models with abundances derived from the published metallicities as a first guess, roughly $5 \times 10^{6} \mathrm{CRAY}-\mathrm{CPU}$ seconds are required, while using converged stratified models with photospheric parameters that are somewhat off the desired ones yields convergence after of the order of $5 \times 10^{5} \mathrm{CPU}$ seconds. Due to this particularly large CPU time demand per model, the convergence criterion for the models was set to be a less than $5 \%$ change in the model flux, which means that the maximal relative correction at any frequency point is no larger than that limit, compared to the result from the previous diffusion iteration step. Only single lines (usually two or three He lines) are actually affected by those larger variations, the continuum flux itself is much more exactly determined and easily complies to our usual " $<10^{-4 "}$ criterion.

The next generation of models will benefit from major improvements of the model atmosphere code and the iteration scheme for the equilibrium condition. Both will reduce the CPU time drastically.

As to the uniqueness of a particular solution, tests indicate that the results agree to within our error limits regardless of the extremely different stratification of diverse start models, making a strong case at least for the stability of the result. We therefore trust that, no matter what the initial conditions in the start model are, the final abundances in the converged model will always be the same.

The emergent fluxes used for comparison with the observations have been calculated from the model atmospheres on a frequency grid optimized for the EUV 
Table 1. Summary of the model atoms. Super-levels (marked by ${ }^{*}$ ) comprise hundreds to thousands of atomic levels. Line transitions between these super-levels (marked by ${ }^{+}$) are composed of all transitions between the individual levels comprising the super-levels (see Werner \& Dreizler 1999 for details).

\begin{tabular}{rcccccccc}
\hline & $\mathrm{H}$ & $\mathrm{He}$ & $\mathrm{C}$ & $\mathrm{N}$ & $\mathrm{O}$ & $\mathrm{Si}$ & $\mathrm{Fe}$ & $\mathrm{Ni}$ \\
\hline non-LTE & & & & & & & & \\
levels & 17 & 54 & 70 & 50 & 24 & 25 & $28^{*}$ & $29^{*}$ \\
lines & 56 & 106 & 218 & 114 & 35 & 53 & $77^{+}$ & $44^{+}$ \\
\hline \hline
\end{tabular}

spectral range. This grid covers $\lambda=50-700 \AA$ in detail and contains more than 30000 frequency points in total.

\subsection{Characteristics of the models}

The models show the general properties as expected from diffusion theory, i.e. the overall trace element abundances decrease with lower $T_{\text {eff }}$ and higher $\log g$ values. Accordingly, the emergent spectra approach the flux distributions of those of pure hydrogen model atmospheres at sufficiently evolved parameters on the cooling sequence. The vertical element distribution however does not generally follow the simple picture of monotonously increasing local abundances with depth, but is strongly affected by the respective radiative acceleration (see Dreizler 1999; Schuh 2000, or Dreizler \& Schuh 2001 for exemplary graphical representations). In particular, gradients may change signs repeatedly, meaning this can be brought about by diffusion processes alone, in contrast to other notions that additionally evoke mass loss effects to explain local abundances that increase towards outer atmospheric regions (e.g. Holberg et al. 1999). In a nutshell, even equilibrium calculations yield rather non-trivial abundance distributions.

As in previous calculations (Dreizler 1999; Dreizler \& Wolff 1999), the iron group element abundance can reach solar values or more, with nickel being at virtually the same level as the iron abundances. Though their dominant effect on the EUV opacity is partly due to the many lines these elements exhibit, it is equally important that their abundances are, over a wide parameter range, simply larger than those of other metals. Of course these two effects are tightly correlated, since more lines directly translate into a stronger radiative acceleration, which in this case easily compensates for the higher atomic weights and thus a stronger response to gravity.

This represents just a short overview of some general effects that can be seen in the models; detailed presentation of depth dependent stratifications for all elements included in the calculations will be published separately.

\section{Model matching: Testing the new models and re-analyzing the DA sample}

To directly compare a theoretical flux distribution with the observed one, it is first re-binned in wavelength and

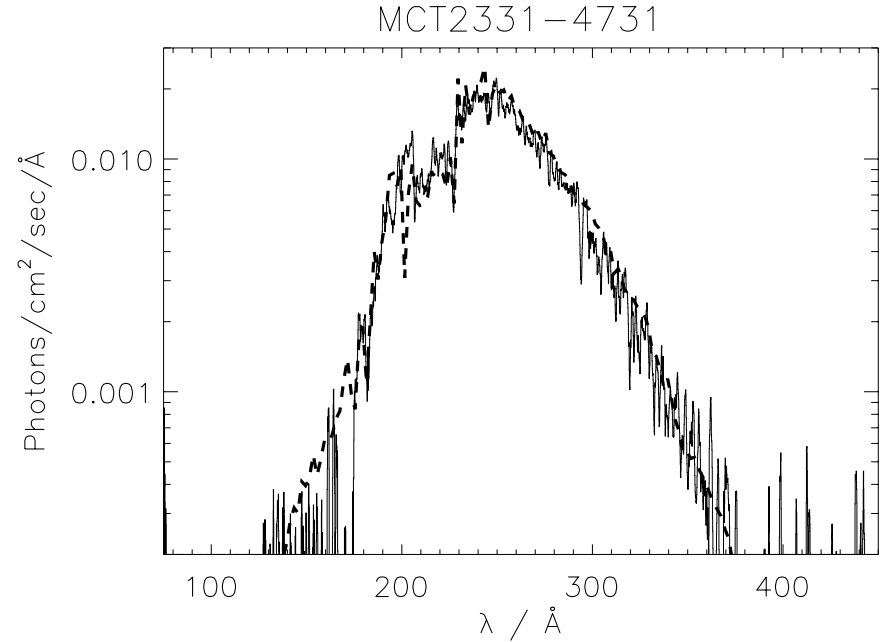

Fig. 1. EUVE spectrum of MCT 2331-4731 with the best-fit model at $T_{\text {eff }}=56000 \mathrm{~K}$ and $\log g=7.7$ overlaid (dashed). The photon flux is displayed on a logarithmic scale.

normalized to the observed visual magnitude. The values for $m_{\mathrm{V}}$ have been chosen using the compilation of McCook \& Sion (1999) and are listed with their individual references in Table 2. Then the effect of interstellar absorption is calculated as sketched in Sect. 2.2.

\subsection{Comparison of theoretical and observed spectra}

The model matching was performed on the mentioned grid which does not in all parameter ranges correspond to a tight mesh, and no interpolation was used. The quality of an individual fit was evaluated by eye.

Of course, changing the interstellar He column densities seemingly has a big impact on the overall run of the spectrum. However, the region most sensitive to metal abundances lies at least partly beyond the He II absorption edge, so once this and the lower energy edges are fitted all remaining deviations have to be due to either a different flux level as caused by the effective temperature or a different amount of absorber content as regulated by the surface gravity in the equilibrium models.

\subsection{Revised atmospheric parameters}

The present model matching led to new atmospheric parameters where mainly the surface gravities differ from former specifications. The improvement of many of the individual fits (see Fig. 2) as compared to fits with homogeneous models (in Wolff et al. 1998; Wolff 1999; Dreizler \& Wolff 1999) vindicates these adjustments.

With homogeneous models, discrepancies were encountered especially for higher metallicity stars in the shorter wavelength regimes, which could not be adjusted by a different metallicity value as this would have deteriorated the quality of the fit at other wavelengths. This difficulty is resolved with the new models, as they offer different abundances at different formation depths. Consequently 

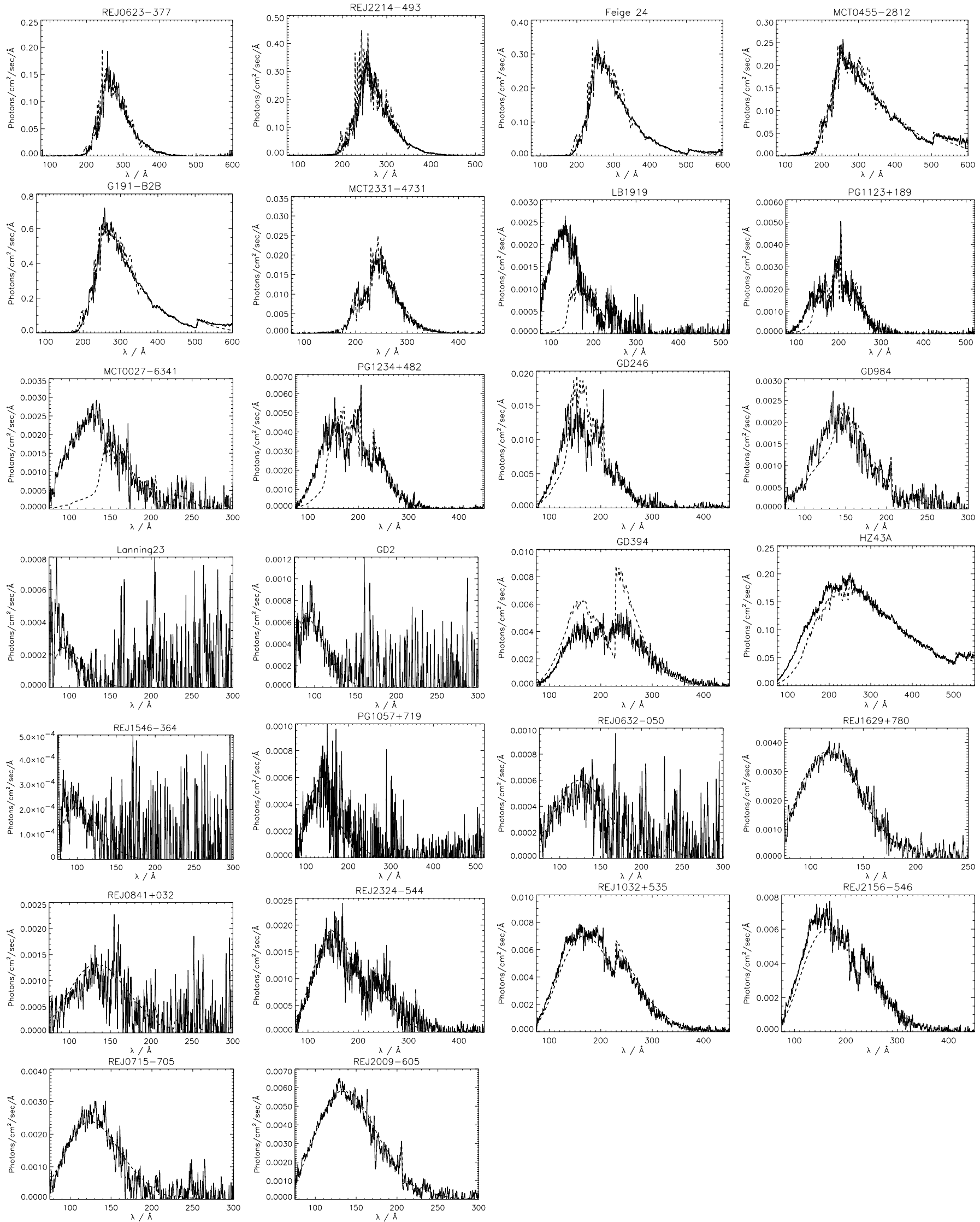

Fig. 2. EUVE spectra of the program stars with theoretical spectra overlaid (dashed), ordered by decreasing metal index $m i$. We only show the spectral range with significant signal. Parameters of the models as well as interstellar column densities for hydrogen and helium can be found in Table 2 . 
Table 2. New and previous results (Wolff et al. 1998) of the EUV analysis ordered by decreasing metal index $m i . N_{\mathrm{H}}$ is given in $10^{18} \mathrm{~cm}^{-2}$. See Sect. 4.3 for an explanation of the theoretical metal index $m i$.

\begin{tabular}{|c|c|c|c|c|c|c|c|c|c|c|c|c|}
\hline \multirow{3}{*}{$\begin{array}{l}{\mathrm{WD}-\mathrm{N}^{\circ}} \\
\text { WD 0621-376 }\end{array}$} & \multirow{3}{*}{$\begin{array}{l}\text { Name } \\
\text { RE J0623-377 }\end{array}$} & \multirow{2}{*}{\multicolumn{2}{|c|}{$m_{\mathrm{V}}$}} & \multirow{3}{*}{$\begin{array}{l}\log g \\
7.2\end{array}$} & \multirow{3}{*}{$\begin{array}{l}T_{\text {eff }} \\
61000\end{array}$} & \multirow{2}{*}{\multicolumn{2}{|c|}{$\begin{array}{l}m i \quad N_{\mathrm{H}} \\
\text { new results }\end{array}$}} & \multirow[t]{2}{*}{$\mathrm{HeI} / \mathrm{H}$} & \multirow[t]{2}{*}{$\mathrm{HeII} / \mathrm{H}$} & \multirow{2}{*}{\multicolumn{3}{|c|}{$\begin{array}{rcc}\log g & T_{\text {eff }} \quad m \\
& \text { previous results }\end{array}$}} \\
\hline & & & & & & & & & & & & \\
\hline & & 12.089 & 1 & & & 3.49 & 5.0 & 0.09 & 0.15 & 7.27 & 58000 & 2.0 \\
\hline WD 2211-495 & RE J2214-493 & 11.7 & 1 & 7.4 & 66000 & 3.02 & 5.8 & 0.07 & 0.15 & 7.38 & 66000 & 4.0 \\
\hline WD $0232+035$ & Feige 24 & 12.56 & 2 & 7.6 & 59000 & 1.22 & 2.72 & 0.068 & 0.25 & 7.17 & 58000 & 1.0 \\
\hline WD 0455-282 & MCT $0455-2812$ & 13.95 & 3 & 7.8 & 66000 & 1.20 & 1.3 & 0.063 & 0.3 & 7.77 & 66000 & 1.0 \\
\hline WD $0501+527$ & G 191-B2B & 11.79 & 2 & 7.6 & 56000 & 0.99 & 2.05 & 0.071 & 0.3 & 7.59 & 56000 & 1.0 \\
\hline WD 2331-475 & MCT 2331-4731 & 13.1 & 4 & 7.6 & 56000 & 0.99 & 8.5 & 0.08 & 0.1 & 8.07 & 56000 & $0.75-1$. \\
\hline WD $1056+516$ & LB 1919 & 16.8 & 4 & 8.2 & 70000 & 0.61 & 16.0 & 0.04 & 0.05 & & 69000 & 0.1 \\
\hline WD $1123+189$ & PG $1123+189$ & 14.13 & 1 & 7.9 & 54000 & 0.43 & 11.9 & 0.09 & 0.052 & 7.63 & 54000 & 0.4 \\
\hline WD 0027-636 & MCT 0027-6341 & 15.0 & 4 & 8.2 & 64000 & 0.42 & 21.5 & 0.068 & 0.06 & 7.96 & 64000 & 0.2 \\
\hline WD $1234+482$ & PG $1234+482$ & 14.38 & 5 & 8.1 & 56000 & 0.31 & 11.7 & 0.09 & 0.05 & 7.67 & 56000 & 0.2 \\
\hline WD $2309+105$ & GD 246 & 13.09 & 2 & 8.2 & 56000 & 0.25 & 18.0 & 0.05 & 0.03 & 7.81 & 59000 & 0.25 \\
\hline WD 0131-164 & GD 984 & 13.98 & 6 & 8.1 & 50000 & 0.20 & 22.0 & 0.068 & 0.035 & 7.67 & 50000 & 0.2 \\
\hline WD $2247+583$ & Lanning 23 & 14.26 & 7 & 8.3 & 56000 & 0.20 & 40.0 & 0.068 & 0.03 & 7.84 & 59000 & 0.25 \\
\hline WD 0004+330 & GD 2 & 13.85 & 2 & 8.25 & 50000 & 0.14 & 82.4 & 0.068 & 0.01 & 7.63 & 49000 & $<0.1$ \\
\hline WD $2111+498$ & GD 394 & 13.09 & 8 & 7.9 & 40000 & 0.13 & 6.5 & 0.07 & 0.15 & 7.94 & 39600 & 0.25 \\
\hline WD $1314+293$ & HZ 43A & 12.914 & 9 & 8.3 & 50000 & 0.13 & 0.9 & 0.06 & 0.0 & 7.99 & 50800 & 0.0 \\
\hline WD 1543-366 & RE J1546-364 & 15.81 & 10 & 8.3 & 50000 & 0.13 & 50.4 & 0.068 & 0.03 & 8.88 & 45200 & 0.0 \\
\hline WD $1057+719$ & PG $1057+719$ & 14.68 & 1 & 8.0 & 42000 & 0.12 & 20.7 & 0.068 & 0.052 & 7.90 & 41500 & 0.1 \\
\hline WD 0630-050 & RE J0632-050 & 15.537 & 1 & 8.2 & 44000 & 0.09 & 30.1 & 0.01 & 0.055 & 8.39 & 44100 & 0.0 \\
\hline WD $1631+781$ & RE J1629+780 & 13.03 & 11 & 8.15 & 42000 & 0.09 & 35.0 & 0.068 & 0.0 & 7.79 & 44600 & 0.05 \\
\hline WD $0838+035$ & RE J0841+032 & 14.48 & 1 & 8.1 & 40000 & 0.08 & 16.0 & 0.068 & 0.052 & 7.78 & 38400 & 0.0 \\
\hline WD $2321-549$ & RE J2324-544 & 15.2 & 1 & 8.2 & 42000 & 0.08 & 9.5 & 0.05 & 0.06 & 7.94 & 45000 & 0.05 \\
\hline WD $1029+537$ & RE J1032+535 & 14.455 & 1 & 8.3 & 44000 & 0.08 & 7.5 & 0.005 & 0.04 & 7.77 & 44000 & 0.0 \\
\hline WD $2152-548$ & RE J2156-546 & 14.44 & 1 & 8.3 & 44000 & 0.08 & 7.0 & 0.04 & 0.04 & 7.91 & 44000 & 0.0 \\
\hline WD $0715-703$ & RE J0715-705 & 14.178 & 1 & 8.3 & 44000 & 0.08 & 21.9 & 0.068 & 0.015 & 8.05 & 44000 & 0.0 \\
\hline WD 2004-605 & RE J2009-605 & 13.6 & 1 & 8.3 & 42000 & 0.06 & 17.5 & 0.06 & 0.015 & 8.16 & 41900 & 0.0 \\
\hline
\end{tabular}

${ }^{1}$ Marsh et al. (1997), ${ }^{2}$ Kidder et al. (1991), ${ }^{3}$ Barstow et al. (1994), ${ }^{4}$ Pounds et al. (1993), ${ }^{5}$ Green (1980), ${ }^{6}$ Wesemael (1995),

${ }^{7}$ Vennes et al. (1997), ${ }^{8}$ Bergeron et al. (1992), ${ }^{9}$ Bohlin et al. (1995), ${ }^{10}$ Vennes et al. (1996b), ${ }^{11}$ Schwartz et al. (1995).

they can often better reproduce especially these formerly delicate regions. Figure 1 shows an example.

Figure 2 displays all observations with the best-fit model spectra over-plotted, both shown at $1 \AA$ resolution. Table 2 lists the parameters of the best-fit model for each object, along with the interstellar column densities used to produce the plots. These are to be regarded as preliminary as fitting improved models to the spectra may yet again yield different values. High He II column densities, found throughout the sample, may be an indication that some opacities are still missing. It additionally lists a quantity denoted $m i$, which stands for the metal index that is to be introduced now.

\subsection{Metal index}

With depth dependent abundances, it is difficult to quote one representative value for each element. To evade this problem, we have introduced a photospheric parameter dependent quantity that relies entirely on the predictions by diffusion theory. As explained above, the absorber content of the models is determined from the equilibrium between the radiative and the gravitational forces. The radiative acceleration scales with $T_{\text {eff }}^{4}$ due to the occurrence of the Eddington flux in the lower expression of Eq. (3), the effective gravitational acceleration scales with $g$ as is evident from the upper expression in the same equation. The absorber content should thus be comparable along lines of constant $T_{\mathrm{eff}}^{4} / g$. To map this ratio onto a dimensionless and easier-to-read parameter, we define

$$
m i=4 \times 10^{-12} \cdot T_{\mathrm{eff}}^{4} / g /\left[\mathrm{K}^{4} \mathrm{~s}^{2} / \mathrm{cm}\right]
$$

where the leading factor has been chosen to yield a result of the order of one for the photospheric parameters of the standard star G191-B2B. Lines of constant mi are displayed in Fig. 3. 


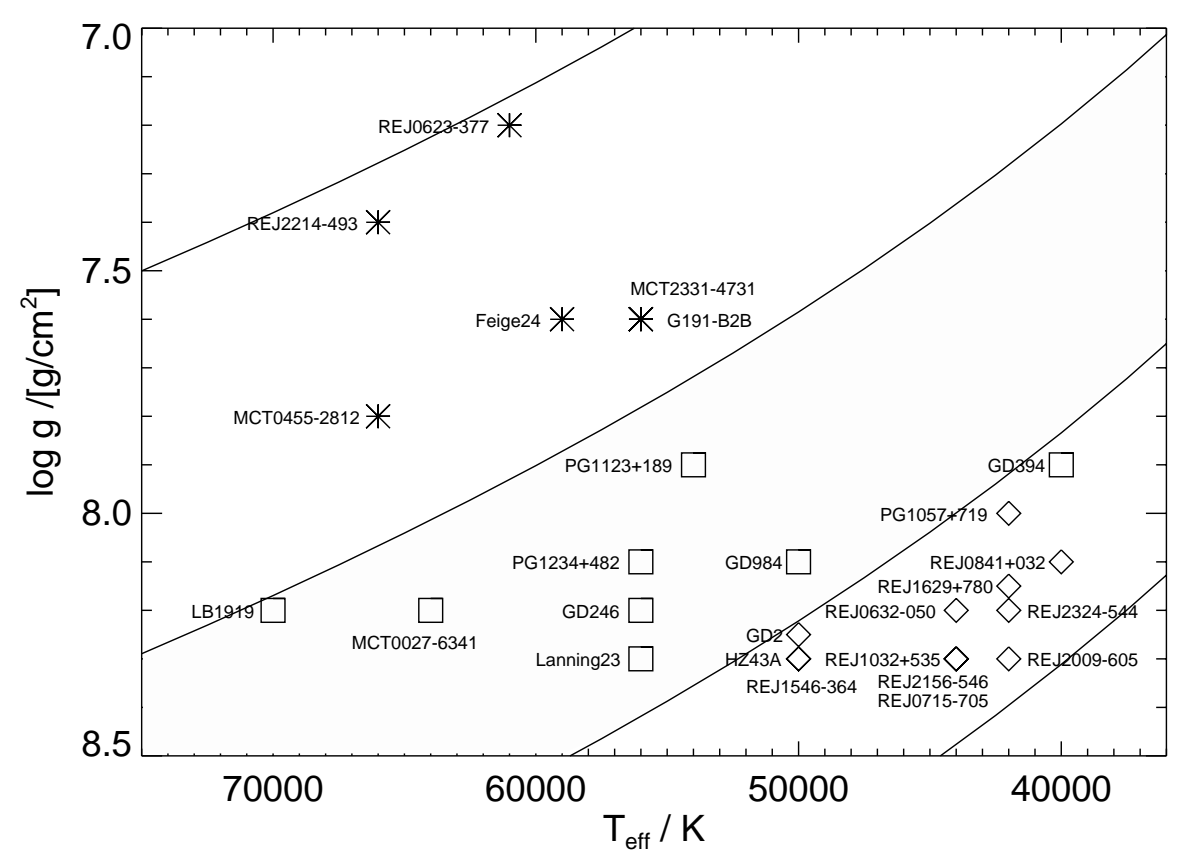

Fig. 3. Revised parameters of the program stars in the $\left[T_{\text {eff }}, \log g\right]$ plane. Lines indicate constant values of the metal index as defined in Sect. 4.3, $m i=4 ., 0.65,0.15,0.05$, respectively. The shaded area indicates the metallicity of DA white dwarfs of the GD 246 group of Wolff et al. (1998). These as well as the objects classified as irregular fall into this regime and are marked by squares. Stars above that region belong to the G 191-B2B-group (identified by starry symbols), stars below to the pure-hydrogen-group (marked by diamond-shaped symbols). See also Sect. 2.1.

It should be emphasized that this quantity is not derived from the actual calculated equilibrium abundances in the models. It is based on a much simpler evaluation of just the same idea that underlies the construction of the models. Knowing this, it is all the more surprising how well the metal indices $m i$ at the newly determined parameters match the metallicities $m$ that Wolff (1999) has derived for the objects.

\section{Discussion}

Our new models can reproduce the observed EUV spectra of hot DA white dwarfs. Since the new models predict the chemical composition from the equilibrium between sedimentation and radiative acceleration, the number of free parameters is drastically reduced to the effective temperature and surface gravity only. The good agreement is a strong evidence that the interplay of these two processes defines the chemical composition and stratification. The agreement is, however, not in all cases better than with chemically homogeneous models, which requires a more detailed discussion.

Stars similar to G 191-B2B (the group one from Wolff et al. 1998) can be reproduced significantly better with our self-consistent stratified models. This result is not surprising since the first and successful application of these models to G 191-B2B (Dreizler \& Wolff 1999) motivated this work in the first place. Our new models can also reproduce the stars classified as pure hydrogen (group three from Wolff et al. 1998), demonstrating that the vanishing of trace amounts of metals is correctly reproduced. Within this group, however, the surface gravity had to be increased by up to 0.5 dex in order to achieve a good fit (see also Table 2).

The fits of PG1123+189, GD 246 and GD 984 are not completely satisfying but deviations are of the same order as compared to fits with homogeneous models. The fits for HZ 43A, REJ1032+535, RE J2156-546 and REJ2009-605 are not quite as good as the ones which could be achieved with spectra derived from the grid of homogeneous models. Further improvements of our models (see Sect. 5.2) may resolve these slight discrepancies.

In four cases (LB 1919, MCT 0027-6341, PG1234+482, and GD 394), using the new models results in a worse fit than before. One may be tempted to explain this by the fact that our model grid is not yet extended enough to find a reasonable agreement. However, significant individual deviations between models and observation could also originate due to physical reasons. As discussed in Sect. 3.1, competing processes can disturb the equilibrium between radiative acceleration and gravitational settling. As is evident from the overall good fit of our models, these are unimportant in general but individual exceptions are possible. In the case of LB 1919 for example, an unusually high rotation rate has been suspected (Finley et al. 1997). Prior to a final decision, we will have to implement several improvements to our models.

\subsection{Remarks on $g$ and $g_{\text {rad }}$}

As mentioned above, our surface gravities are in several cases higher than in earlier analyses. Those analyses had 
evaluated the Balmer lines for a determination of the surface gravity. Should the surface gravities determined with our models prove to be reliable, they would open up a new possibility: the surface gravity governs the gravitational settling. Through the equilibrium condition, the amount of trace metals in the atmosphere is determined. In effect, we therefore evaluate the metal abundances in order to derive the surface gravity. The new models with stratified metal abundances can modify the Balmer lines via the changed atmospheric structure. This could explain the deviations in the surface gravity determination. A more detailed investigation is in progress. Our proposed method would be a very sensitive indicator but it is, unfortunately, also sensitive to systematic errors in the calculation of the radiative acceleration. The shifts in log $g$ necessary to obtain good fits for several of the objects consequently could indicate that the accuracy of the $g_{\text {rad }}$ calculation needs to be further improved. The next generation of models will therefore aim at determining radiative accelerations with higher precision (see Sect. 5.2).

One independent result, however, might be worth considering in this context: detailed parallax and gravitational redshift measurements with HST of the binary system Feige 24 (Benedict et al. 2000; Vennes et al. 2000) also yielded a higher surface gravity for the WD component than several optical analyses $(\log g=7.2-7.5)$. The consistency of our result ( $\log g=7.7)$ with Vennes et al.'s (log $g=7.5-7.7)$ might be interpreted as a hint that our current results do not suffer from an inaccurate $g_{\text {rad }}$ determination in the models after all. Instead, analyses with homogeneous models would have been biased due to systematic errors introduced through the neglect of stratification. But even though this may sound plausible, it is too early to call such a statement anything more than a suspicion.

\subsection{Conclusions and perspective}

In this paper, we have presented our chemically stratified model atmospheres describing the chemical composition through the equilibrium between gravitational settling and radiative acceleration self-consistently. The application to 26 EUVE spectra of hot DA white dwarfs has revealed an overall good agreement, demonstrating the potential of these new models.

In upcoming papers, we will check the derived parameters through a re-analysis of the Balmer lines and of the metal lines observable in UV spectra. The analysis of optical spectra with our new stratified models should allow to check for systematic error as compared to previous analyses with chemically homogeneous models. This will also be an important test for the consistency of atmospheric parameters derived from different parts of the spectrum, which has often posed a problem in the past, and to which we hope the new type of models will ultimately be a solution. Only this test will show on how a firm ground the parameters presented here stand. Likewise, the determination of the interstellar column densities has to be regarded as preliminary, since these cannot be determined independently from the theoretical spectra.

The current models are only a first step, in the future we will be able to present an improved set of stratified model atmospheres. A "next generation" version of our stellar atmosphere code itself as well as a more efficient coupling between the computation of the model structure and the chemical stratification will allow to include much more detailed model atoms and at the same time many more chemical elements. This influences and is crucial for the accuracy of the calculation of the radiative acceleration. By evaluating the scale of the deviation in comparison to the current results it should then become clear how trustworthy the latter are.

Acknowledgements. The authors would like to thank Klaus Werner (Tübingen) for useful comments and discussions. We also thank Detlev Koester (Kiel) who provided part of the source code for the handling of the ISM absorption. The referee has provided very valuable comments to help improving this paper. All model atmospheres have been calculated on CRAY machines of the Rechenzentrum der Universität Kiel. This work is supported by the Deutsche Forschungsgemeinschaft under grant DR 281/13-1.

\section{References}

Barstow, M. A., Fleming, T. A., Diamond, C. J., et al. 1993, MNRAS, 264, 16

Barstow, M. A., Wesemael, F., Holberg, J. B., et al. 1994, MNRAS, 267, 647

Barstow, M. A., Dobbie, P. D., Holberg, J. B., Hubeny, I., \& Lanz, T. 1997, MNRAS, 286, 58

Barstow, M. A., Hubeny, I., \& Holberg, J. B. 1999, MNRAS, 307,884

Benedict, G. F., McArthur, B. E., Franz, O. G., et al. 2000, AJ, 119, 2382

Bergeron, P., Saffer, R. A., \& Liebert, J. 1992, ApJ, 394, 228

Bohlin, R. C., Colina, L., \& Finley, D. S. 1995, AJ, 110, 1316

Chayer, P., Fontaine, G., \& Wesemael, F. 1995a, ApJS, 99, 189

Chayer, P., Vennes, S., Pradhan, A. K., et al. 1995b, ApJ, 454, 429

Dreizler, S. 1999, A\&A, 352, 632

Dreizler, S. \& Wolff, B. 1999, A\&A, 348, 189

Dreizler, S., \& Schuh, S. 2001, in ASP Conf. Ser. 226, The 12th European Workshop on White Dwarfs, ed. J. L. Provençal, H. L. Shipman, J. MacDonald, \& S. Goodchild, 69

Dupuis, J., Chayer, P., Vennes, S., Christian, D. J., \& Kruk, J. W. 2000, ApJ, 537, 977

Finley, D. S., Koester, D., \& Basri, G. 1997, ApJ, 488, 375

Fontaine, G., \& Michaud, G. 1979, ApJ, 231, 826

Green, R. F. 1980, ApJ, 238, 685

Hearn, D. R., Richardson, J. A., Bradt, H. V. D., et al. 1976, ApJ, 203, L21

Heber, U., Napiwotzki, R., \& Reid, I. N. 1997, A\&A, 323, 819

Holberg, J. B., Barstow, M. A., Bruhweiler, F. C., Hubeny, I., \& Green, E. M. 1999, ApJ, 517, 850

Jordan, S., Koester, D., Wulf-Mathies, C., \& Brunner, H. 1987, A\&A, 185, 253

Jordan, S., Wolff, B., Koester, D., \& Napiwotzki, R. 1994, A\&A, 290, 834 
Kahn, S. M., Wesemael, F., Liebert, J., et al. 1984, ApJ, 278, 255

Kidder, K. M., Holberg, J. B., \& Mason, P. A. 1991, AJ, 101, 579

Koester, D. 1989, in IAU Colloq. 114: White Dwarfs, ed. G. Wegner, Lecture Notes in Physics, 328, 206

Koester, D., Dreizler, S., Weidemann, V., \& Allard, N. F. 1998, A\&A, 338, 612

Lampton, M., Margon, B., Paresce, F., Stern, R., \& Bowyer, S. 1976, ApJ, 203, L71

MacDonald, J. 1992, ApJ, 394, 619

Margon, B., Malina, R., Bowyer, S., Cruddace, R., \& Lampton, M. 1976, ApJ, 203, L25

Marsh, M. C., Barstow, M. A., Buckley, et al. 1997, MNRAS, 286,369

McCook, G. P., \& Sion, E. M. 1999, ApJS, 121, 1

Mewe, R., Heise, J., Gronenschild, E. H. B. M., et al. 1975, ApJ, 202, L67

Paerels, F. B. S., \& Heise, J. 1989, ApJ, 339, 1000

Paquette, C., Pelletier, C., Fontaine, G., \& Michaud, G. 1986, ApJS, 61, 177

Petre, R., Shipman, H. L., \& Canizares, C. R. 1986, ApJ, 304, 356

Pounds, K. A., Allan, D. J., Barber, C., et al. 1993, MNRAS, 260, 77

Rumph, T., Bowyer, S., \& Vennes, S. 1994, AJ, 107, 2108

Schatzman, E. 1949, Publ. Københavns Obs., No. 149
Schuh, S. 2000, Diploma Thesis, Eberhard-Karls-Universität Tübingen

Schwartz, R. D., Dawkins, D., Findley, D., \& Chen, D. 1995, PASP, 107, 667

Shipman, H. L. 1976, ApJ, 206, L67

Unglaub, K., \& Bues, I. 1998, A\&A, 338, 75

Vauclair, G., Vauclair, S., \& Greenstein, J. L. 1979, A\&A, 80, 79

Vennes, S., Chayer, P., Fontaine, G., \& Wesemael, F. 1989, ApJ, 336, L25

Vennes, S., Chayer, P., Hurwitz, M., \& Bowyer, S. 1996a, ApJ, 468,898

Vennes, S., Thejll, P. A., Wickramasinghe, D. T., \& Bessell, M. S. 1996b, ApJ, 467, 782

Vennes, S., Thejll, P. A., Galvan, R. G., \& Dupuis, J. 1997, ApJ, 480, 714

Vennes, S., Polomski, E. F., Lanz, T., et al. 2000, ApJ, 544, 423

Werner, K., \& Dreizler, S. 1999, in Computational Astrophysics, ed. H. Riffert, \& K. Werner, vol. 109 (Amsterdam: Elsevier Press), 65

Wesemael, F. 1995, in preparation

Wolff, B. 1999, Ph.D. Thesis, Christian-Albrechts-Universität Kiel

Wolff, B., Jordan, S., \& Koester, D. 1996, A\&A, 307, 149

Wolff, B., Koester, D., Dreizler, S., \& Haas, S. 1998, A\&A, 329, 1045 